\title{
Fabrication and Mode Coupling of Long-Period Fiber Grating by Winding a Wire Around an Optical Fiber Fixed to a Cylindrical Metal Rod
}

\author{
Jonghun Lee, Cherl-Hee Lee, Kwang Taek Kim and Jaehee Park
}

Additional information is available at the end of the chapter

http://dx.doi.org/10.5772/53069

\section{Introduction}

A long-period fiber grating (LPFG) couple light from the fundamental guided core mode to co-propagating cladding modes at specific resonance wavelengths. A LPFG can be fabricated by induction of periodic modulations of the refractive index along the core of a single-mode optical fiber. The pitch of a typical LPFG ranges from $100 \mu \mathrm{m}$ to $1000 \mu \mathrm{m}$, which is larger than that of a fiber Bragg gratings (FBGs) by more than two orders of magnitude. The transmission spectrum of a LPFG consists of a number of rejection bands at the resonance wavelengths, like as a band-rejection filter. LPFGs were first proposed and demonstrated by Vengsarkar and others [1] as band-rejection filters, due to their high sensitivity as well as low insertion loss and return loss, LPFGs are becoming more and more popular as simple and versatile optical components for mode converters [2], gain flattening filters [3], and fiber-optic sensors [4]. Many methods have been demonstrated for the fabrication of LPFGs. An ultra-violet (UV) writing method, which causes the index change of a photosensitive Ge-doped silica fiber core by UV light radiation, was successfully applied to the writing of LPFGs [5]. The amount of index change depends on the intensity and the duration of the UV laser. Because of the symmetrically-formed index modulation in the core, only axially symmetric cladding modes are coupled in a UV-written LPFG. Many non-UV methods have also been demonstrated for the fabrication of LPFGs, such as the electric-discharge writing method [6], divided coil heaters method [7], microbending method [8-9], and mechanically induced method [10-11-12]. However, all of these methods involve expensive fabrication equipments or additional fixed devices, thereby increasing the installation costs. Thus, the inexpensive manufacture of small LPFGs has been major barrier for the realization of practical optical communication systems. Recently, winding wires has been presented as a new fabrication method to form LPFGs on an optical fiber [13-14]. In 
$[13,14]$, since the coupling wavelength of the grating is defined by the period of the v-grooved tube, this requires very difficult and delicate fabrication operations. Thus, a new simple and flexible fabrication process was presented for the fabrication of low-cost LPFGs that does not require any formation of periodic grooves [15]. The wire-winding long-period fiber grating (WW-LPFG) presented in [15] is based on the periodic winding of a wire around an optical fiber attached to a cylindrical metal rod without any grooves. The periodic pressure from the winding wire on an optical fiber creates a periodic change in the refractive index of the optical fiber core, and makes mechanically-induced LPFGs on the optical fiber. A fabricated WWLPFG with high tension of the winding wire on the optical fiber induces a high index variation on the fiber core. Thus, the coupling coefficient of the WW-LPFG was calculated according to the refractive index variation of the fiber core. The new approach is presented for the easy fabrication of wire-winding long-period gratings (WW-LPFGs) based on the periodic winding a wire around an optical fiber attached to a cylindrical rod. The periodic pressure of the wire induces change of the refractive index of the optical fiber core; thus, resonance wavelengths of the grating can be easily controlled according to the winding-wire pitch controlled by a microprocessor. At the winding-wire pitch of $500 \mu \mathrm{m}$, the spectra show resonance-wavelength dips corresponding to the $\mathrm{LP}_{02}, \mathrm{LP}_{03}, \mathrm{LP}_{04}$, and $\mathrm{LP}_{05}$ cladding modes; the resonance wavelengths go to longer wavelengths at the increased the winding pitch of $510 \mu \mathrm{m}$. These are in good agreement with theoretical values. The polarization dependence of a resonance wavelength shift of $9 \mathrm{~nm}$ and transmission power difference of $2.5 \mathrm{~dB}$ was shown at the 520 $\mu \mathrm{m}$ grating pitch. When the diameter of the cylindrical metal rod was smaller, or higher tension from the winding pressure was applied, stronger mode coupling resulted in deeper dips in the transmission spectra according to the induced higher index variation.

\section{Properties of long-period fiber grating}

\subsection{Effective index}

The electric field in a cylindrical coordinate system has three independent vector components as a function of $r, \phi$, and $\mathrm{z}$ :

$$
E(r, \phi, z)=\hat{r} E_{r}(r, \phi, z)+\hat{\phi} E_{\phi}(r, \phi, z)+\hat{z} E_{z}(r, \phi, z) .
$$

Unlike the $\mathrm{E}_{\mathrm{r}}$ and $\mathrm{E}_{\phi}$ component, the $\mathrm{E}_{z}$ component does not couple to the other components of $E_{r}$ and $E_{\phi}$; therefore, the scalar wave equation for $E_{z}$ can exist independently and easily be solved. Thus, the other fields, $E_{r}$ and $E_{\phi}$ are derived easily through the calculated $E_{z}$ component. The wave equation for the $\mathrm{z}$ component of the field vectors is given as follows in the homogeneous region:

$$
\left(\nabla^{2}+k^{2}\right)\left\{\begin{array}{l}
E_{z} \\
H_{z}
\end{array}\right\}=0,
$$

where $\mathrm{k}^{2}=\omega^{2} \mu \varepsilon$ and $\nabla^{2}$ is the Laplacian operator in the cylindrical coordinate given by 


$$
\nabla^{2}=\frac{\partial^{2}}{\partial r^{2}}+\frac{1}{r} \frac{\partial}{\partial r}+\frac{1}{r^{2}} \frac{\partial^{2}}{\partial \phi^{2}}+\frac{\partial^{2}}{\partial z^{2}},
$$

Every component of the fields, including $E_{z}$, is assumed to have the angular frequency $\omega$ and the wavenumber of $\beta$ to the longitudinal direction z-axis, and Eqn. (2) can be written as

$$
\begin{aligned}
& \frac{\partial^{2} E_{z}}{\partial r^{2}}+\frac{1}{r} \frac{\partial E_{z}}{\partial r}+\frac{1}{r^{2}} \frac{\partial^{2} E_{z}}{\partial \phi^{2}}+\left[k^{2} n(r, \phi)^{2}-\beta^{2}\right] E_{z}=0 \\
& \frac{\partial^{2} H_{z}}{\partial r^{2}}+\frac{1}{r} \frac{\partial H_{z}}{\partial r}+\frac{1}{r^{2}} \frac{\partial^{2} H_{z}}{\partial \phi^{2}}+\left[k^{2} n(r, \phi)^{2}-\beta^{2}\right] H_{z}=0
\end{aligned}
$$

where $n(r, \phi)$ is the refractive index of an optical fiber at the transverse axis. Here, $\mathrm{E}_{z}$ and $\mathrm{H}_{z}$ can be expressed with the form of the Bessel differential function of order $l, l=0,1,2,3, \ldots$, so $\mathrm{E}_{z}$ and $\mathrm{H}_{z}$ are single-valued function of $\phi$ :

$$
\begin{aligned}
& E_{z}=\left\{\begin{array}{l}
A J_{l}(\kappa r) e^{j l \phi} e^{-j \beta z},(0 \leq r \leq a) \\
C K_{l}(\gamma r) e^{j l \phi} e^{-j \beta z},(r>a)
\end{array}\right. \\
& H_{z}=\left\{\begin{array}{l}
B J_{l}(\kappa r) e^{j l \phi} e^{-j \beta z},(0 \leq r \leq a) \\
D K_{l}(\gamma r) e^{j l \phi} e^{-j \beta z},(r>a)
\end{array}\right.
\end{aligned}
$$

At Maxwell's curl equations, Eqn. (6), the transverse electromagnetic fields are related to $E_{z}$ and $\mathrm{H}_{\mathrm{z}}$ :

$$
\nabla \times E=-j \omega \mu H, \nabla \times H=j \omega \varepsilon E,
$$

Substituing Eqn. (5) to Eqn. (6), all the transverse components are obtained as follows:

At the core region $(\mathrm{r}<\mathrm{a})$ :

$$
\begin{aligned}
& E_{r}=-\frac{j \beta}{\kappa^{2}}\left[A \kappa J_{l}^{\prime}(\kappa r)+B \frac{j \omega \mu l}{\beta r} J_{j}(\kappa r)\right] e^{j l \phi} e^{-j \beta z} \\
& E_{\phi}=-\frac{j \beta}{\kappa^{2}}\left[A \frac{j l}{r} J_{l}(\kappa r)-B \frac{\omega \mu}{\beta} \kappa J_{l}^{\prime}(\kappa r)\right] e^{j l \phi} e^{-j \beta z} \\
& H_{r}=-\frac{j \beta}{\kappa^{2}}\left[-A \frac{j \omega \varepsilon_{c o} l}{\beta r} J_{l}(\kappa r)+B \kappa J_{l}^{\prime}(\kappa r)\right] e^{j l \phi} e^{-j \beta z} \\
& H_{\phi}=-\frac{j \beta}{\kappa^{2}}\left[A \frac{\omega \varepsilon_{c o}}{\beta} \kappa J_{l}^{\prime}(\kappa r)+B \frac{j l}{r} J_{l}(\kappa r)\right] e^{j l \phi} e^{-j \beta z}
\end{aligned}
$$

At the cladding region $(r>a)$ : 


$$
\begin{aligned}
& E_{r}=\frac{j \beta}{\gamma^{2}}\left[C \gamma K_{l}^{\prime}(\gamma r)+D \frac{j \omega \mu l}{\beta r} K_{j}(\gamma r)\right] e^{j l \phi} e^{-j \beta z} \\
& E_{\phi}=\frac{j \beta}{\gamma^{2}}\left[C \frac{j l}{r} K_{l}(\gamma r)-D \frac{\omega \mu}{\beta} \gamma K_{l}^{\prime}(\gamma r)\right] e^{j l \phi} e^{-j \beta z} \\
& H_{r}=\frac{j \beta}{\gamma^{2}}\left[-C \frac{j \omega \varepsilon_{c l} l}{\beta r} K_{l}(\gamma r)+D \gamma K_{l}^{\prime}(\gamma r)\right] e^{j l \phi} e^{-j \beta z} \\
& H_{\phi}=\frac{j \beta}{\gamma^{2}}\left[C \frac{\omega \varepsilon_{c l}}{\beta} \gamma K_{l}^{\prime}(\gamma r)+D \frac{j l}{r} K_{l}(\gamma r)\right] e^{j l \phi} e^{-j \beta z}
\end{aligned}
$$

where $\varepsilon_{\mathrm{co}}=\varepsilon_{0} \mathrm{n}_{\mathrm{co}^{2}}, \varepsilon_{\mathrm{cl}}=\varepsilon_{0} \mathrm{n}_{\mathrm{cl}}{ }^{2}$, and

$$
J_{l}^{\prime}(\kappa r)=\frac{d J_{l}(\kappa r)}{d(\kappa r)}, K_{l}^{\prime}(\gamma r)=\frac{d K_{l}(\gamma r)}{d(\gamma r)}
$$

The boundary condition that $\mathrm{E}_{\mathrm{z}}$ and $\mathrm{H}_{\mathrm{z}}$ should be continuous at $\mathrm{r}=\mathrm{a}$ leads to

$$
C=A \frac{J_{l}(\kappa a)}{K_{l}(\gamma a)}, D=B \frac{J_{l}(\kappa a)}{K_{l}(\gamma a)}
$$

The boundary condition that $\mathrm{E}_{\phi}$ should be continuous at $\mathrm{r}=\mathrm{a}$ leads to

$$
A \frac{j l}{a}\left(\frac{1}{\kappa^{2}}+\frac{1}{\gamma^{2}}\right)=B \frac{\omega \mu}{\beta}\left[\frac{1}{\kappa} \frac{J_{l}^{\prime}(\kappa a)}{J_{l}(\kappa a)}+\frac{1}{\gamma} \frac{K_{l}^{\prime}(\gamma a)}{K_{l}(\gamma a)}\right]
$$

The boundary condition that $\mathrm{H}_{\phi}$ should be continuous at $\mathrm{r}=\mathrm{a}$ leads to

$$
A\left[\frac{\omega \varepsilon_{c o}}{\beta} \frac{1}{\kappa} \frac{J_{l}^{\prime}(\kappa a)}{J_{l}(\kappa a)}+\frac{\omega \varepsilon_{c l}}{\beta} \frac{1}{\gamma} \frac{K_{l}^{\prime}(\gamma a)}{K_{l}(\gamma a)}\right]=-B \frac{j l}{a}\left(\frac{1}{\kappa^{2}}+\frac{1}{\gamma^{2}}\right) .
$$

Equation (11) and (12) yield the following equation determining the effective index and propagation constant:

$$
\frac{\beta^{2} l^{2}}{a^{2}}\left(\frac{1}{\kappa^{2}}+\frac{1}{\gamma^{2}}\right)^{2}=\left(\frac{1}{\kappa} \frac{J_{l}^{\prime}(\kappa a)}{J_{l}(\kappa a)}+\frac{1}{\gamma} \frac{K_{l}^{\prime}(\gamma a)}{K_{l}(\gamma a)}\right)\left(k_{0}^{2} n_{c o}^{2} \frac{1}{\kappa} \frac{J_{l}^{\prime}(\kappa a)}{J_{l}(\kappa a)}+k_{0}^{2} n_{c l}^{2} \frac{1}{\gamma} \frac{K_{l}^{\prime}(\gamma a)}{K_{l}(\gamma a)}\right) .
$$

If weakly guiding condition, $\mathrm{n}_{\mathrm{co}} \cong \mathrm{n}_{\mathrm{cl}}$, Eqn. (13) is simplified to

$$
n_{e f f}^{2} \frac{l^{2}}{a^{2}}\left(\frac{1}{\kappa^{2}}+\frac{1}{\gamma^{2}}\right)^{2}=\left(\frac{1}{\kappa} \frac{J_{l}^{\prime}(\kappa a)}{J_{l}(\kappa a)}+\frac{1}{\gamma} \frac{K_{l}^{\prime}(\gamma a)}{K_{l}(\gamma a)}\right)^{2}
$$

By using the Bessel function identity, 


$$
\frac{J_{l}^{\prime}(\kappa a)}{\kappa J_{l}(\kappa a)}= \pm \frac{J_{l \mp 1}(\kappa a)}{\kappa J_{l}(\kappa a)} \mp \frac{l}{\kappa^{2}}, \frac{K_{l}^{\prime}(\gamma a)}{\gamma K_{l}(\gamma a)}=\mp \frac{K_{l \mp 1}(\gamma a)}{\gamma K_{l}(\gamma a)} \mp \frac{l}{\gamma^{2}},
$$

we can get

$$
\left( \pm \frac{J_{l \mp 1}(\kappa a)}{\kappa a J_{l}(\kappa a)} \mp \frac{l}{(k a)^{2}}\right)+\left(\mp \frac{K_{l \mp 1}}{\gamma a K_{l}} \mp \frac{l}{(\gamma a)^{2}}\right)= \pm l\left[\frac{1}{(\kappa a)^{2}}+\frac{1}{(\gamma a)^{2}}\right] .
$$

From Eqn. (16) two different equations corresponding to the two values of $+l,-l$ are obtained and the eigenvalues resulting from these two equations yield the two classes of solutions designated as the EH modes and HE modes. The characteristic equations of the EH and HE modes correspond to the upper sign and the lower sign, respectively.

For EH mode:

$$
\frac{1}{\kappa a} \frac{J_{l+1}(\kappa a)}{J_{l}(\kappa a)}=-\frac{1}{\gamma a} \frac{K_{l+1}(\gamma a)}{K_{l}(\gamma a)}
$$

For HE mode:

$$
\begin{aligned}
& \frac{1}{\kappa a} \frac{J_{0}(\kappa a)}{J_{1}(\kappa a)}=\frac{1}{\gamma a} \frac{K_{0}(\gamma a)}{K_{1}(\gamma a)},(l=1) \\
& \frac{1}{\kappa a} \frac{J_{l-1}(\kappa a)}{J_{l-2}(\kappa a)}=-\frac{1}{\gamma a} \frac{K_{l-1}(\gamma a)}{K_{l-2}(\gamma a)},(l \geq 2)
\end{aligned}
$$

Some characteristic equations among the TE, TM, EH, and HE modes are the same under the weakly guiding approximation, and the degenerate modes are classified as approximated linear polized $(\mathrm{LP} l m)$ modes:

$$
\frac{1}{\kappa a} \frac{J_{l}(\kappa a)}{J_{l-1}(\kappa a)}=-\frac{1}{\gamma a} \frac{K_{l}(\gamma a)}{K_{l-1}(\gamma a)} .
$$

Figure 1 shows a graphical determination of the effective index of $\mathrm{LP} \mathrm{P}_{01}$ core mode and Fig. 2 shows the mode intensity of LP01 core mode. The cladding modes can be obtained by applying the boundary continuity conditions at the core-cladding boundary and the cladding-air boundary. Erdogan [16] proposed an accurate description of the mode propagation in the cladding, in which the cladding modes are not approximated as being linearly polarized. Applying the core-cladding boundary condition, the characteristic equation for a cladding mode was given by

$$
\zeta_{0}=\zeta_{0}^{\prime}
$$




$$
\begin{gathered}
\zeta_{0}=\frac{1}{\sigma_{2}} \frac{u_{2}\left(J K+\frac{\sigma_{1} \sigma_{2} u_{21} u_{32}}{n_{2}^{2} a_{1} a_{2}}\right) p_{l}\left(a_{2}\right)-K q_{l}\left(a_{2}\right)+J r_{l}\left(a_{2}\right)-\frac{1}{u_{2}} s_{l}\left(a_{2}\right)}{-u_{2}\left(\frac{u_{32}}{n_{2}^{2} a_{2}} J-\frac{u_{21}}{n_{1}^{2} a_{1}} K\right) p_{l}\left(a_{2}\right)+\frac{u_{32}}{n_{1}^{2} a_{2}} q_{l}\left(a_{2}\right)+\frac{u_{21}}{n_{1}^{2} a_{1}} r_{l}\left(a_{2}\right)} \\
\zeta_{0}^{\prime}=\sigma_{1} \frac{u_{2}\left(\frac{u_{32}}{a_{2}} J-\frac{n_{3}^{2} u_{21}}{n_{2}^{2} a_{1}} K\right) p_{l}\left(a_{2}\right)+\frac{u_{32}}{a_{2}} q_{l}\left(a_{2}\right)+\frac{u_{21}}{a_{1}} r_{l}\left(a_{2}\right)}{u_{2}\left(\frac{n_{3}^{2}}{n_{2}^{2}} J K+\frac{\sigma_{1} \sigma_{2} u_{21} u_{32}}{n_{1}^{2} a_{1} a_{2}}\right) p_{l}\left(a_{2}\right)-\frac{n_{3}^{2}}{n_{1}^{2}} K q_{l}\left(a_{2}\right)+J r_{l}\left(a_{2}\right)-\frac{n_{2}^{2}}{n_{1}^{2} u_{2}} s_{l}\left(a_{2}\right)}
\end{gathered}
$$

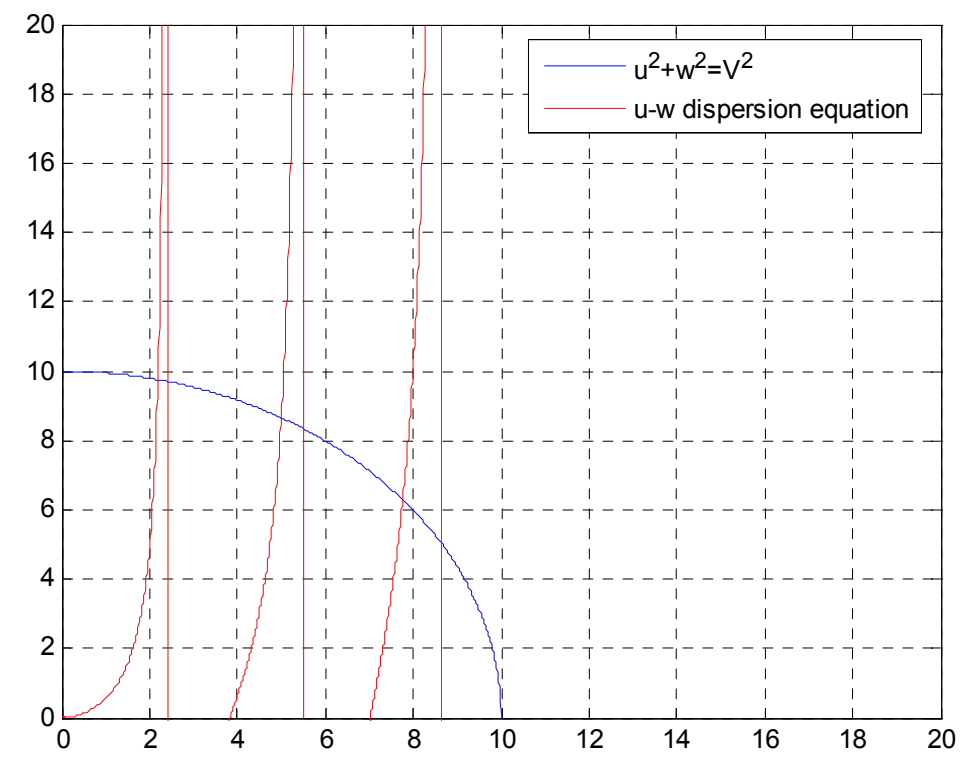

Figure 1. Graphical determination of the effective index of core mode.

Figure 3 shows the graphical determination of the effective indice of cladding modes according to Eqn. (20), which are determined by the crossing points of the left-hand side equation and the right-hand side equation. 


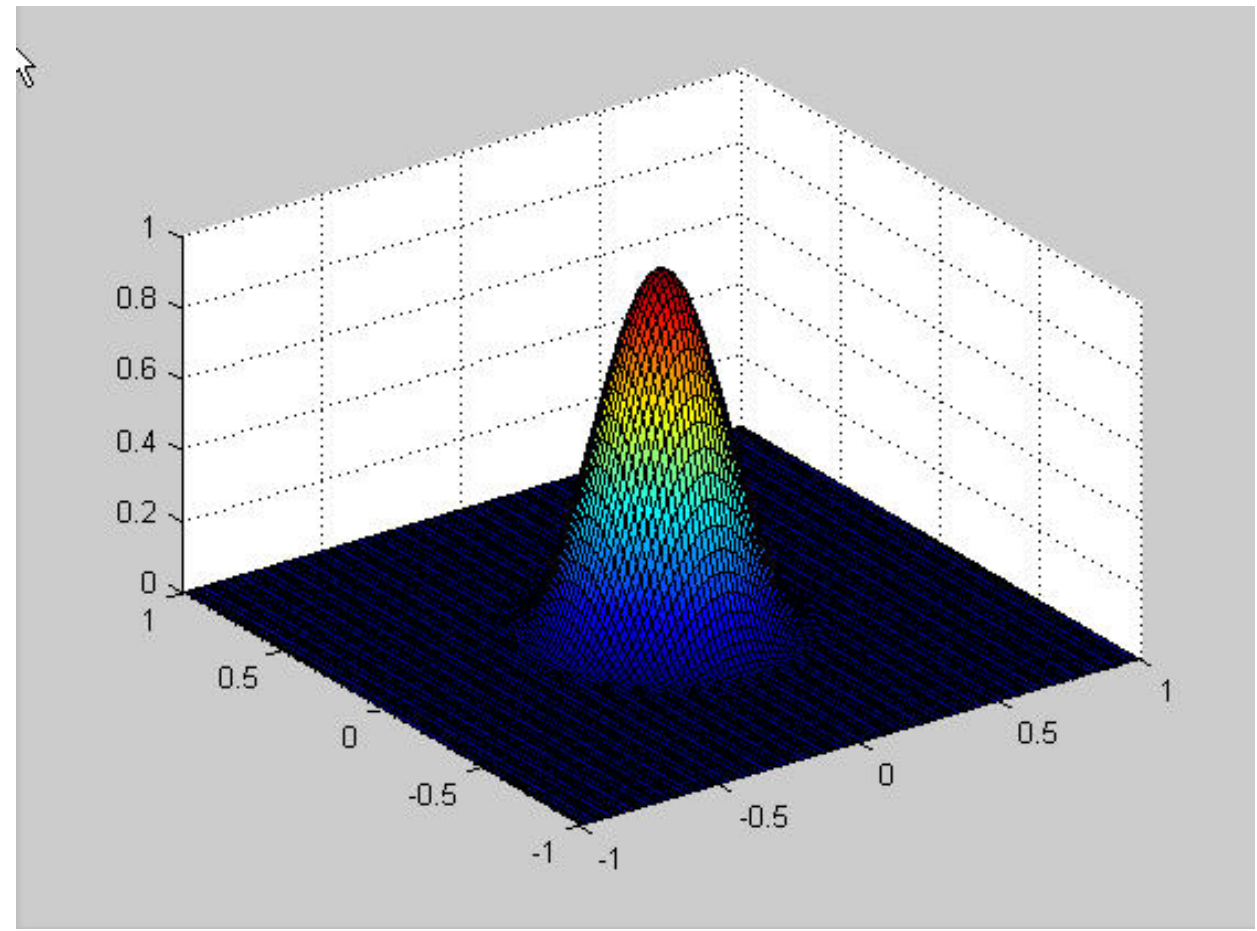

Figure 2. Mode intensity distribution of LP01 core mode

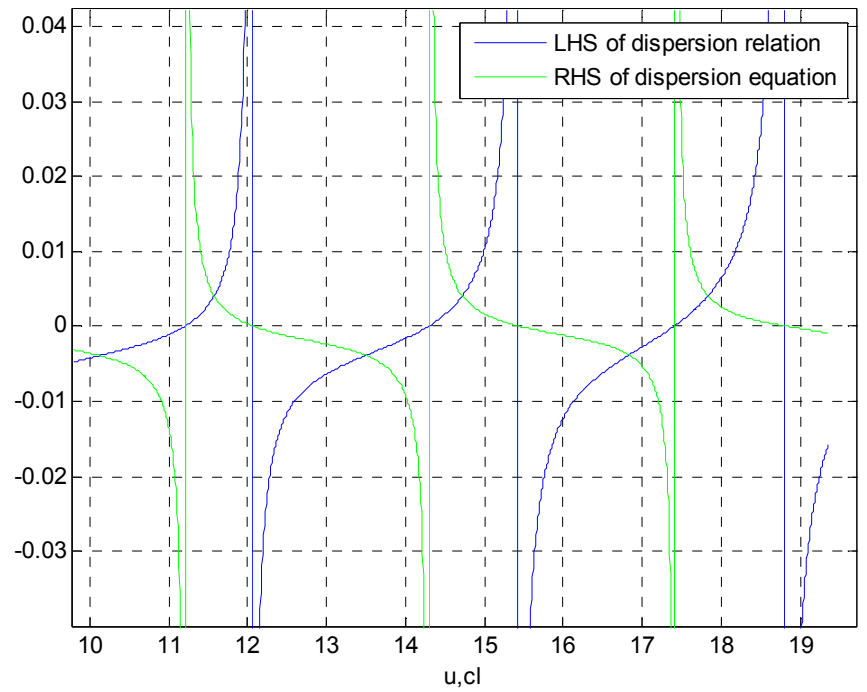

Figure 3. Graphical determination of the effective index of cladding modes. 


\subsection{Coupling coefficient}

The transverse electric fields are described by a linear superposition of forward-propagating and backward-propagating guided modes of the unperturbed optical fiber:

$$
E(x, y, z)=\left[A^{+}(z) e^{-j \beta z}+A^{-}(z) e^{j \beta z}\right] e_{t}(x, y)
$$

where $\mathrm{A}^{+}(\mathrm{z})$ and $\mathrm{A}^{-}(\mathrm{z})$ are slowly varying amplitudes traveling in the $+\mathrm{z}$ and $-\mathrm{z}$ directions, respectively; $\beta$ is the propagation constant; and $e_{t}(x, y)$ is the transverse mode field. The mode coupling of the electric field occurs at the gratings of the optical fiber core, which acts as a perturbation in the refractive index. Thus, the coupled mode equation can be written as

$$
\begin{aligned}
& \frac{d A^{+}}{d z}=-j A^{+} K \mathrm{e}^{-j\left(\beta^{+}-\beta^{+}\right) z}-j A^{-} K \mathrm{e}^{j\left(\beta^{+}+\beta^{-}\right) z} \\
& \frac{d A^{-}}{d z}=j A^{+} K \mathrm{e}^{-j\left(\beta^{+}+\beta^{-}\right) z}+j A^{-} K \mathrm{e}^{j\left(\beta^{-}-\beta^{-}\right) z}
\end{aligned}
$$

where $\mathrm{K}(\mathrm{z})$, the general transverse coupling coefficient between modes, is given

$$
\begin{aligned}
K(z) & =\frac{\omega}{4} \int_{-\infty}^{\infty} \int_{-\infty}^{\infty} \Delta \varepsilon(x, y, z) e_{t}(x, y) \cdot e_{t}^{*}(x, y) d x d y \\
& =\frac{\omega}{4} \int_{-\infty}^{\infty} \int_{-\infty}^{\infty}\left\{2 n_{c o} \delta n_{c o}\left[1+v \cos \left(\frac{2 \pi}{\Lambda} z+\phi\right)\right]\right\}_{t}(x, y) \cdot e_{t}^{*}(x, y) d x d y \\
& =\frac{\omega n_{c o}}{2} \int_{-\infty}^{\infty} \int_{-\infty}^{\infty} \delta n_{c o} e_{t}(x, y) \cdot e_{t}^{*}(x, y) d x d y+\frac{\omega n_{c o}}{2} \frac{v}{2} \int_{-\infty}^{\infty} \int_{-\infty}^{\infty} 2 \cos \left(\frac{2 \pi}{\Lambda} z+\phi\right) e_{t}(x, y) \cdot e_{t}^{*}(x, y) d x d y \\
& =\frac{\omega n_{c o}}{2} \int_{-\infty}^{\infty} \int_{-\infty}^{\infty} \delta n_{c o} e_{t}(x, y) \cdot e_{t}^{*}(x, y) d x d y+\frac{v}{2}\left[\frac{\omega n_{c o}}{2} \int_{-\infty}^{\infty} \int_{-\infty}^{\infty} e_{t}(x, y) \cdot e_{t}^{*}(x, y) d x d y\right]\left[2 \cos \left(\frac{2 \pi}{\Lambda} z+\phi\right)\right] \\
& =\sigma(z)+\left[\frac{v}{2} \sigma(z)\right]\left[2 \cos \left(\frac{2 \pi}{\Lambda} z+\phi\right)\right] \\
& =\sigma(z)+\kappa\left[2 \cos \left(\frac{2 \pi}{\Lambda} z+\phi\right)\right]
\end{aligned}
$$

where $\Delta \varepsilon$ is the permittivity variation of a fiber core and $v$ is the fringe visibility associated with the index change. When two new coefficients are defined,

$$
\begin{aligned}
& \sigma(z)=\frac{\omega n_{c 0}}{2} \overline{\delta n}_{c o} \int_{-\infty}^{\infty} \int_{-\infty}^{\infty} e_{t}(x, y) \cdot e_{t}^{*}(x, y) d x d y \\
& \kappa(z)=\frac{\omega n_{c o} v}{4} \overline{\delta n}_{c o} \int_{-\infty}^{\infty} \int_{-\infty}^{\infty} \Delta \varepsilon(x, y, z) e_{t}(x, y) \cdot e_{t}^{*}(x, y) d x d y,
\end{aligned}
$$

where $\sigma$ is a dc coupling coefficient and $\kappa$ is an ac coupling coefficient. By substituting Eqn. (25),(26) to Eqn. (24), the electric fields in the grating can be simplified to the superposition of forward-propagating and backward-propagating fundamental modes in the optical fiber core: 


$$
\begin{aligned}
& \frac{d R(z)}{d z}=i \hat{\sigma}(z) R(z)+i \kappa(z) S(z) \\
& \frac{d S(z)}{d z}=-i \hat{\sigma}(z) S(z)-i \kappa^{*}(z) R(z),
\end{aligned}
$$

where $R(z)=A^{+}(z) \exp [i(\delta z-\phi / 2)]$ and $S(z)=A^{-}(z) \exp [-i(\delta z+\phi / 2)]$. The general dc self-coupling coefficient can be represented by

$$
\hat{\sigma}=\delta+\sigma-\frac{1}{2} \frac{d \phi}{d z}=\left(\beta-\frac{\pi}{\Lambda}\right)+\sigma-\frac{1}{2} \frac{d \phi}{d z}
$$

where $\delta$ is the detuning factor, the $\phi$ and $\mathrm{d} \phi / \mathrm{dz}$ are the grating phase and possible chirp of the grating period, respectively. The solution of the complex reflection and transmission coefficient can be

$$
\begin{aligned}
& R(z)=\frac{\gamma \cosh [\gamma(z-L / 2)]+i \hat{\sigma} \sinh [\gamma(z-L / 2)]}{\gamma \cosh (\gamma L)-i \hat{\sigma} \sinh (\gamma L)} \\
& S(z)=\frac{-i \kappa \sinh [\gamma(z-L / 2)]}{\gamma \cosh (\gamma L)-i \hat{\sigma} \sinh (\gamma L)}
\end{aligned}
$$

where $\gamma$ is described by

$$
\begin{aligned}
& \gamma=\sqrt{\kappa^{2}-\hat{\sigma}^{2}},\left(\text { if }, \kappa^{2}>\hat{\sigma}^{2}\right) \\
& \gamma=i \sqrt{\hat{\sigma}^{2}-\kappa^{2}},\left(\text { if }, \kappa^{2}<\hat{\sigma}^{2}\right) .
\end{aligned}
$$

The amplitude reflection coefficients can then be shown to be

$$
\rho=\frac{S(-L / 2)}{R(-L / 2)}=\frac{-\kappa \sinh (\gamma L)}{\hat{\sigma} \sinh (\gamma L)+\gamma \cosh (\gamma L)} .
$$

\subsection{Resonance wavelength}

With the knowledge of the core and cladding effective indices, it is possible to determine LPFG resonance wavelengths for a specific grating period. This can be achieved by the phase matching condition in [16]. The transmission spectrum has dips at the wavelengths corresponding to resonances with various cladding modes. The resonance wavelength satisfying the phase-matching condition is given by [17]

$$
\lambda_{0}=\left(n_{e f f, c o}^{01}-n_{e f f, c l}^{0 m}\right) \Lambda
$$

where $\Lambda$ is the period of the grating, and neff,co ${ }^{01}$ and neff,cl ${ }^{0 m}$ are the effective indices of the LP01 core mode and $\mathrm{LP}_{0 m}$ cladding modes at the resonance wavelength, respectively. Based on the phase-matched condition, the relationship between the period of the grating period $(\Lambda)$ 
and resonance wavelength $\left(\lambda_{0}\right)$ can be obtained by the calculation of the propagation constants of the LP01 core and various cladding modes of a fiber at each specific wavelength. Figure 4 shows the resonance wavelength versus grating period characteristic for coupling with five orders of cladding modes, and for a specific grating pitch the spectrum yields resonance wavelength values for all visible bands.

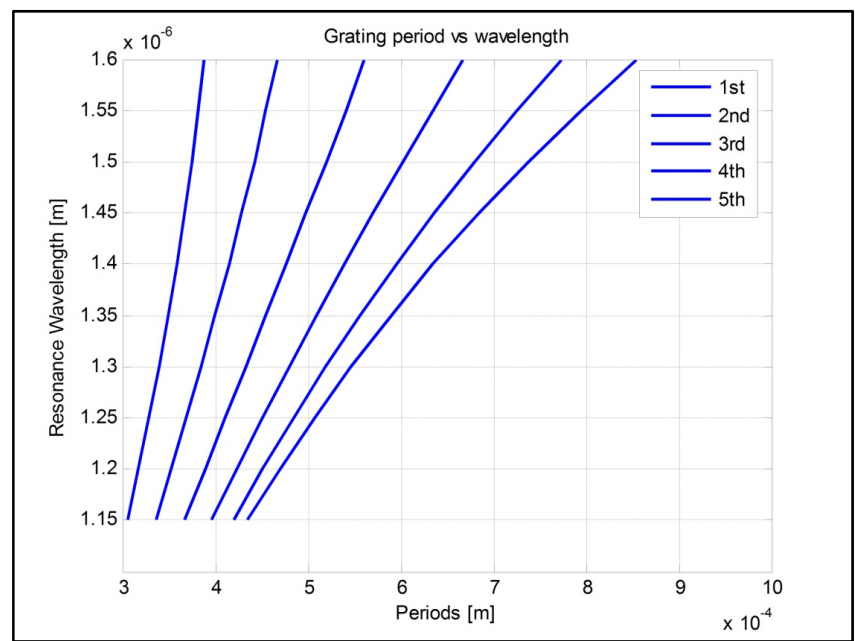

Figure 4. Resonance wavelength versus grating period.

Coupling mode theory applied to the three-layer model yields an expression for the corecladding mode coupling coefficient by Erdogan [16]. The transmission spectrum modelled as $\Lambda=455 \mu \mathrm{m}, \mathrm{L}=20 \mathrm{~mm}$, and $\Delta \mathrm{n}_{\mathrm{co}}=1 \times 10^{-4}$ is shown in Fig. 5 .

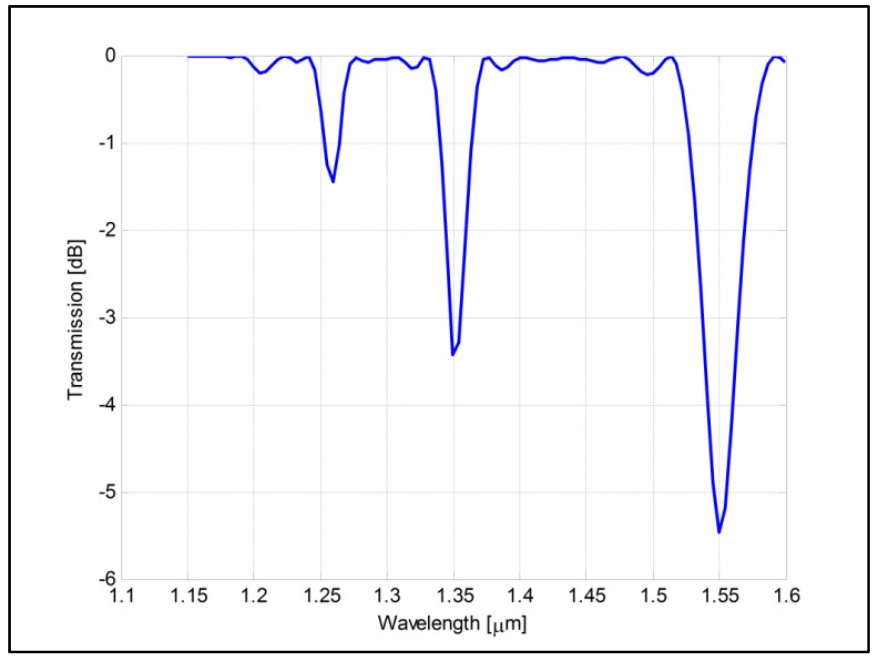

Figure 5. Transmission spectrum 


\section{Experiments and results}

A LPFG has coupling characterisitics according to the phase-matching condition between a core mode and cladding modes in an optical fiber. A forward-propagating core mode (LP01 mode) is coupled to forward-propagating cladding modes ( $\mathrm{LP} l m$ mode) of the fiber. While LPFG generally allows coupling of a core mode to all order cladding modes ( $\mathrm{LP}_{l m}$ mode), untilted WW-LPFG couples a core mode to only $l=0$ order cladding modes ( $\mathrm{LP}_{0} m$ mode). The proposed WW-LPFG behaving as an un-tilted LPFG couples a core mode to LPom modes.

Figure 6 shows the structure and photo of the manufactured WW-LPFG. The SMF-28 standard fiber with a numerical aperture (NA) of 0.14 , refractive index difference of $0.36 \%$, core and cladding diameters of 8.2 and $125 \mu \mathrm{m}$, respectively, was used. The refractive indices of core and cladding were 1.4489 and 1.444, respectively, at a $1550 \mathrm{~nm}$ wavelength. A copper wire with the diameter of $170 \mu \mathrm{m}$ was used as a winding wire. The jacket of a SMF was stripped away to allow a copper wire to be wound around the fiber. The SMF was then attached to a cylindrical metal rod with a $10 \mathrm{~mm}$ diameter.

Figure 7 shows the apparatus and manufacturing process to fabricate the WW-LPFG. After an optical fiber was attached to the cylindrical metal rod, one end of a copper wire was glued to the cylindrical metal rod, and the other end of the copper wire was pulled by the mass of $900 \mathrm{~g}$ weight at the angle $(\theta)$ of $60^{\circ}$, convertible to the tension of $8.8 \mathrm{~N}$. When the cylindrical metal rod was rotated by a microprocessor, the wire holder controlled by a microprocessor is moved to the longitudinal direction of the cylindrical metal rod according to the designed pitch, so that the optical fiber was wound by the wire within the desired region. The lateral stress of the wire periodically pressing on the optical fiber changes the refractive index of the optical fiber, so the pitch of the winding wire is the key factor to the controlling of the resonance wavelength of the WW-LPFG. The experimental setup consisted of a broadband light source (Agilent, 83437A), the fabricated WW-LPFG, and an optical spectrum analyzer (Ando, AQ6315A). The light from the broadband light source propagated in the WW-LPFG, and the power intensity characteristics of the transmitted light were then determined when it reached the OSA.

Figure 8 shows the relationship of resonance wavelength with the grating period for the coupling of fundamental core mode to cladding modes. The transmission spectrum has dips at the wavelengths corresponding to resonances with various cladding modes. The resonance wavelength satisfying the phase-matching condition is given by Eqn. (32). Based on the phase-matched condition, the relationship between the period of the grating period and the resonance wavelength can be obtained by the calculation of the propagation constants of the LP01 core and various cladding modes of a fiber at each specific wavelength. Resonance wavelengths where guided-to-cladding mode coupling takes place can be theoretically determined by proper selection of a specific grating period. The vertical dashed line at the grating period of $500 \mu \mathrm{m}$ meets with the wavelengths of $1280 \mathrm{~nm}, 1330 \mathrm{~nm}, 1380$ $\mathrm{nm}$, and $1490 \mathrm{~nm}$ of $\mathrm{LP}_{02}, \mathrm{LP}_{03}, \mathrm{LP}_{04}, \mathrm{LP}_{05}$ modes, respectively. The resonance wavelengths of $\mathrm{LP}_{02}$, LP03, LP04, LP05 modes correspond to $1280 \mathrm{~nm}, 1330 \mathrm{~nm}, 1380 \mathrm{~nm}$, and $1490 \mathrm{~nm}$, 
respectively. The parameters of the winding pitch, number of turns, and winding length of the copper wire are $500 \mu \mathrm{m}, 40$ turns, and $20 \mathrm{~mm}$, respectively. Figure 9 shows the experimental and simulated transmission spectra of the WW-LPFG under the same conditions as those in Fig. 8. The simulated transmission spectra calculated by simulation software, OptiGrating (Optiwave Systems Inc) were in good agreement with the experimental result at the resonance wavelengths and transmission power loss. At $1490 \mathrm{~nm}$, the transmission power gain of the resonance wavelength was $-7.7 \mathrm{~dB}$ and the full width at half maximum (FWHM) was $20 \mathrm{~nm}$.
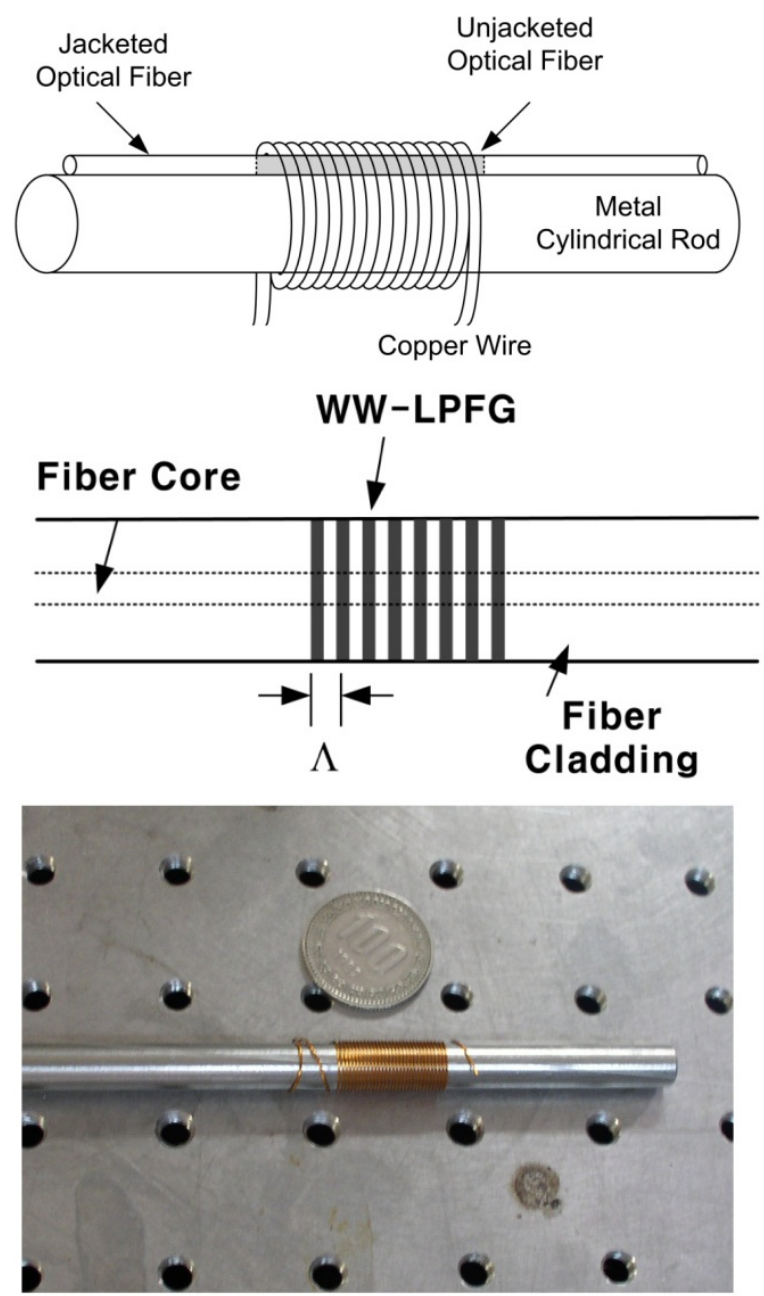

Figure 6. Structure of the WW-LPFG. 

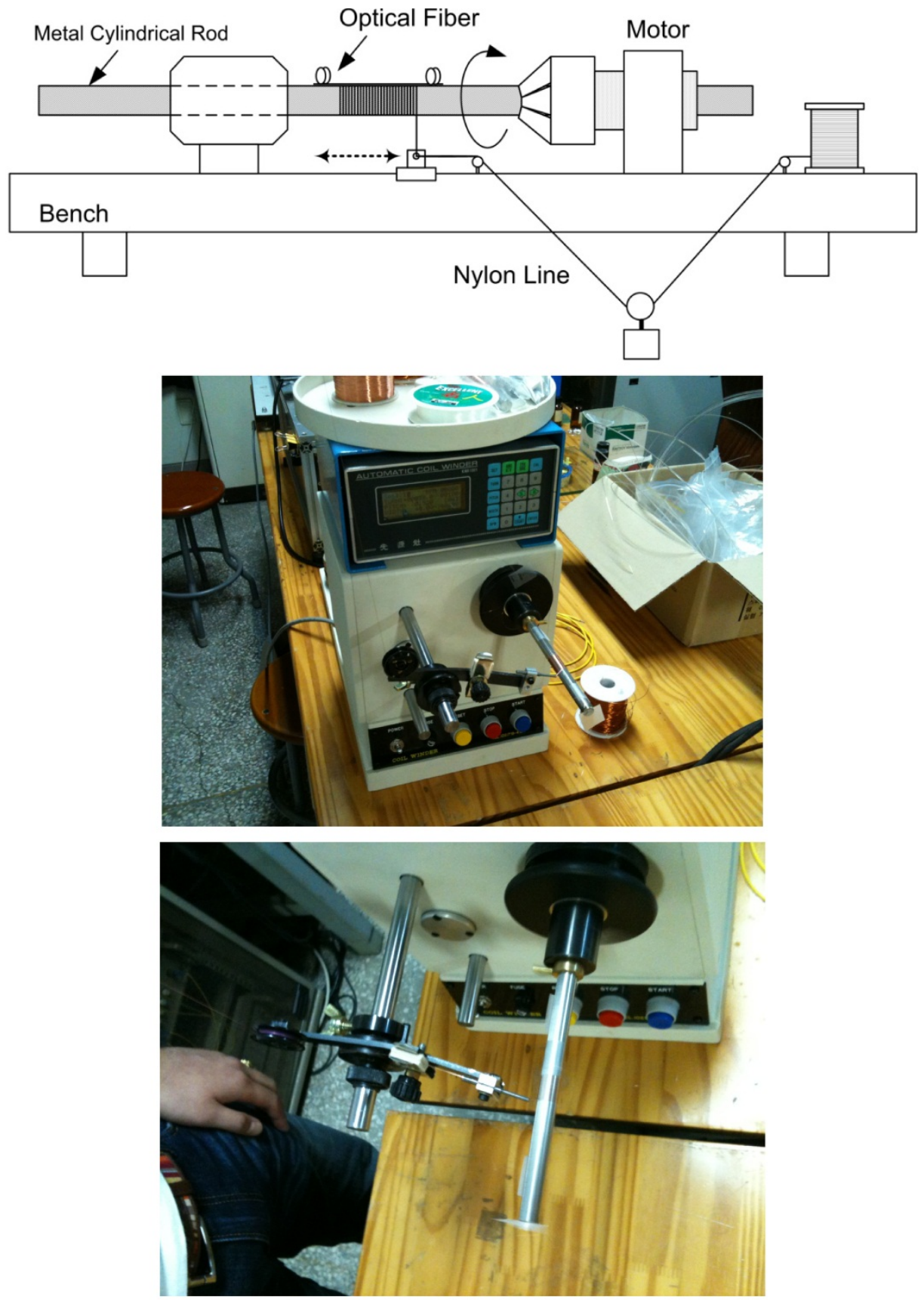

Figure 7. Experimental setup and apparatus 


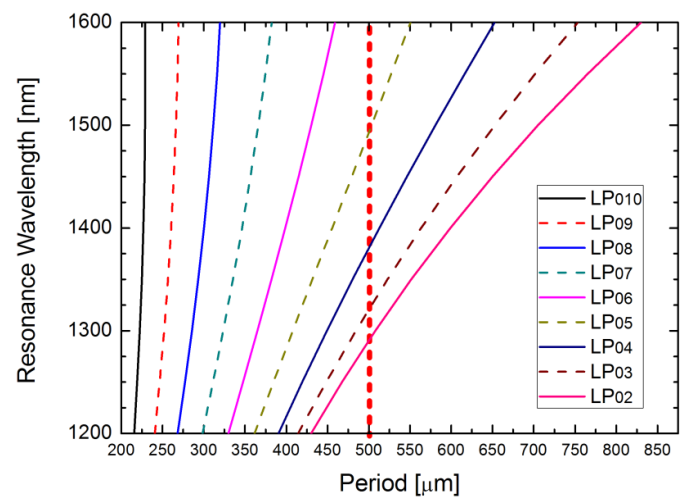

Figure 8. Resonance wavelength versus grating period.

\section{Simulation :}
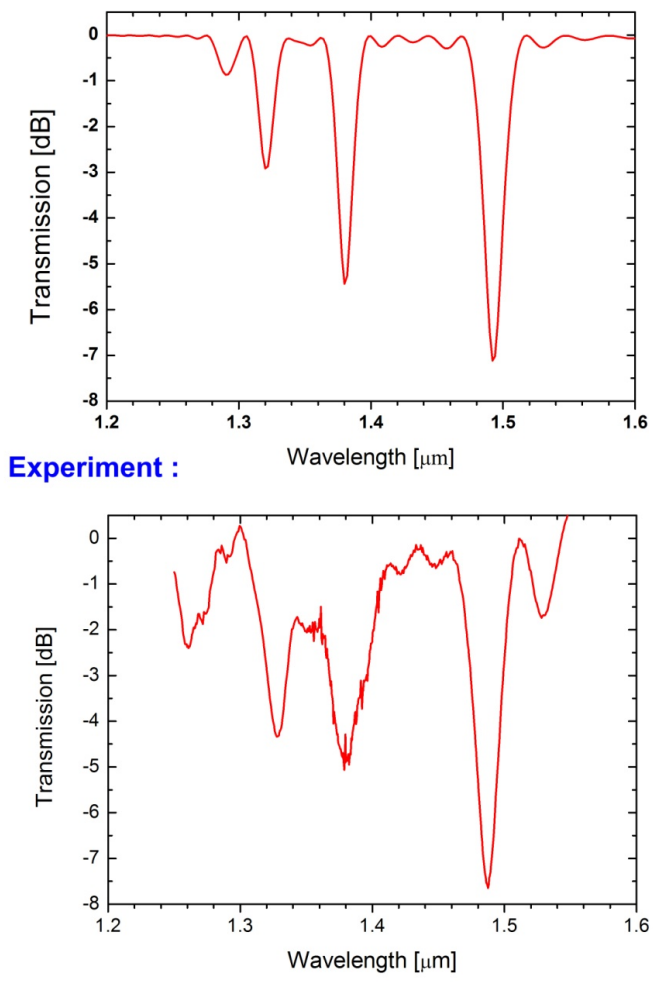

Figure 9. Transmission spectrum at the grating pitch of 500um

The spectral dependence of the power transmission of the LP01 mode in the LPFG can be determined by [16] 


$$
\frac{P_{01}(L)}{P_{01}(0)}=\cos ^{2}\left(\sqrt{\kappa^{2}+\delta^{2}} L\right)+\frac{1}{1+\frac{\kappa^{2}}{\delta^{2}}} \sin ^{2}\left(\sqrt{\kappa^{2}+\delta^{2}} L\right)
$$

where $\delta$ is the detuning parameter

$$
\delta=\frac{1}{2}\left\{\beta_{c o}^{01}-\beta_{c l}^{0 m}-\frac{2 \pi}{\Lambda}\right\},
$$

$\kappa$ is the coupling constant for the grating, $\mathrm{L}$ is the grating length, and $\beta_{\mathrm{co}}{ }^{01}$ and $\beta_{\mathrm{cl}}{ }^{0 \mathrm{~m}}$ are the propagation constants of the $\mathrm{LP}_{01}$ core mode and $\mathrm{LP}_{0 m}$ cladding modes, respectively. The standard silica optical fiber is a cylindrical and isotropic dielectric waveguide with a circularly symmetric refractive index distribution. However, as the applied tension of the wire induces deformations of the optical fiber, the refractive indices parallel and perpendicular to the direction of the applied tension are differently changed and the birefringence is derived from the photo-elastic property of the fiber. The refractive index changes at any point of the fiber are [18]

for x-polarization,

$$
\left(\Delta n_{e f f}\right)_{x}=-\frac{1}{2}\left(n_{e f f, 0}\right)^{3}\left[P_{11} \varepsilon_{x}+P_{12}\left(\varepsilon_{y}+\varepsilon_{z}\right)\right]
$$

for y-polarization,

$$
\left(\Delta n_{e f f}\right)_{y}=-\frac{1}{2}\left(n_{e f f, 0}\right)^{3}\left[P_{11} \varepsilon_{y}+P_{12}\left(\varepsilon_{x}+\varepsilon_{z}\right)\right] .
$$

Here, neff, 0 is the initial effective refractive index of the core of unperturbed fiber, $\mathrm{P}_{11}$ and $\mathrm{P}_{12}$ are the photo-elastic coefficients of the unperturbed optical fiber, and $\varepsilon_{x}, \varepsilon_{y}, \varepsilon_{z}$ are the strain components at the fiber in the $\mathrm{x}, \mathrm{y}$, and $\mathrm{z}$ direction, respectively. Because of the mechanical and photo-elastic properties of the optical fiber's material $\left(\mathrm{P}_{11}<\mathrm{P}_{12}, \varepsilon_{x}>0, \varepsilon_{y}<0, \varepsilon_{x}<\varepsilon_{y}\right)$, the refractive index change of the $x$-polarization is higher than that of the y-polarization. Since the circular fiber of the fabricated WW-LPFG is periodically pressed in one direction to form a mechanically induced long-period grating, birefringence due to the asymmetric structure occurs.

As shown in Fig. 10, the spectrum of the fabricated WW-LPFG has polarization dependence on $\mathrm{x}$ - and y-polarization lights. When an LP01 core mode is coupled to an LP04 cladding mode at a $520 \mu \mathrm{m}$ grating pitch, the resonant wavelengths of $\mathrm{x}$ - and y-polarization light appeared at 1435 and $1426 \mathrm{~nm}$, respectively. By the birefringence effect, the polarization dependence makes the change of $9 \mathrm{~nm}$ resonance wavelength and $2.5 \mathrm{~dB}$ transmitted light power. Figure 11 shows the transmission spectra of the LP04 mode of the WW-LPFG with the pitch of $500 \mu \mathrm{m}$ and with several masses of $0.6,0.9$, and $1.2 \mathrm{~kg}$ weight, convertible to the tension of $5.8,8.8$, and $11.7 \mathrm{~N}$, respectively. The higher tension by the winding wire pressure induced the higher index variation, which resulted in stronger mode coupling and thus generally in deeper dips in the transmission spectra. Meanwhile, the resonant wavelength did not shift by the change of the applied tension. 
The transmission spectra of the $\mathrm{LP}_{04}$ mode of the WW-LPFG with the diameters of the cylindrical metal rod of 7 and $10 \mathrm{~mm}$ were shown in Fig. 12 at the fixed pitch of $500 \mu \mathrm{m}$. When the diameter of the cylindrical metal rod is smaller, the pressing region on the fiber attached to the cylindrical metal rod is wider, and thus the higher index variation of the fiber results in deeper dips in the transmission spectrum.

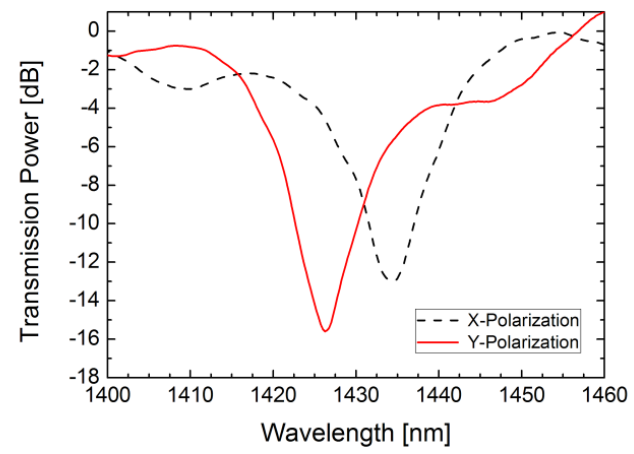

Figure 10. Polarization dependence of an $\mathrm{LP} 04$ mode at a grating pitch of 520um

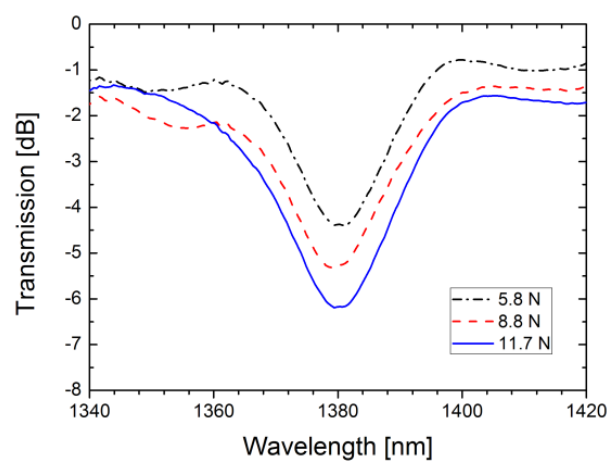

Figure 11. Transmission spectra of the WW-LPFG with different wire tensions.

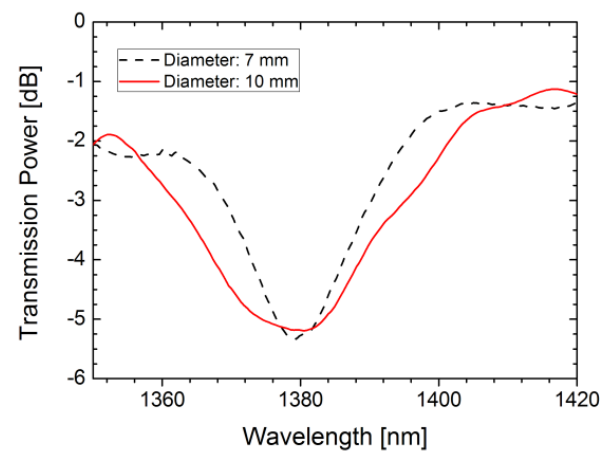

Figure 12. Transmission spectra of the WW-LPFG with different diameters of the cylindrical metal rod. 


\section{Conclusion}

An easily fabricated WW-LPFG is wire-wound periodically around an optical fiber attached to a cylindrical rod. The periodic pressure of the wire results in refractive index change of an optical fiber core, and the resonance wavelengths of the grating can be easily controlled according to the winding-wire pitch controlled by a microprocessor. Due to the simple and flexible fabrication process using the winding of a wire, the fabricated WW-LPFG is small, and cost-effective, and the resonance wavelength are easily controlled by the pitch of the winding-wire using a microprocessor. At the winding-wire pitch of $500 \mu \mathrm{m}$ and $510 \mu \mathrm{m}$, the spectra show resonance-wavelength dips corresponding to the $\mathrm{LP}_{02}, \mathrm{LP}_{03}, \mathrm{LP}_{04}, \mathrm{LP} 05$ cladding modes. The polarization dependence of a resonance wavelength shift of $9 \mathrm{~nm}$ was shown. Smaller diameter of the cylindrical metal rod and higher tension of the winding wire led to the stronger mode coupling according to induced higher index variation. With smaller diameter of the cylindrical metal rod, or higher tension due to the winding pressure, stronger mode coupling can result in deeper dips in the transmission spectra according to induced higher index variation.

\section{Author details}

Jonghun Lee and Cherl-Hee Lee

Robotics Research Division, Daegu Gyeongbuk Institute of Science \& Technology,

Daegy, Korea

Kwang Taek Kim

Department of Optoelectronics, Honam University, Gwangju, Korea

Jaehee Park

Department of Electronic Engineering, Keimyung University, Daegu, Korea

\section{Acknowledgement}

This work was supported by the DGIST R\&D Program of the Ministry of Education, Science and Technology of Korea(12-RS-02).

\section{References}

[1] Vengsarkar A M, Lemaire J P, Judkins J B, Bhatia V, Erdogan T, Sipe J E. Long-period Fiber Gratings as Band-rejection Filters. Journal of Lightwave Technology 1996; 14: 5865.

[2] Ostling D, Engan H E. Broadband Spatial Mode Conversion by Chirped Fiber Bending. Optics Letter 1996; 21: 192-194.

[3] Vengsarkar A M, Pedrazzani J R, Judkins J B, Lemaire J P, Bergano N S, Davidson C R. Long Period Fiber Grating Based Gain Equalizers. Optics Letter 1996; 21: 336-338. 
[4] Bhatia V, Vengsarkar A M. Optical Fiber Long Period Grating Sensors. Optics Letter 1996; 21: 692-694.

[5] Martin J, Ouellette F. Novel Written Technique of Long and Highly Reflection In-fiber Gratings. Electron Letters 1994; 30: 811-812.

[6] Humbert G, Malki A. Annealing Time Dependence at Very High Temperature of Electric Arc-induced Long-period Fibre Gratings. Electron Letters 2002; 38: 449-450.

[7] Bae J K, Kim S H, Kim J H, Bae J, Lee S B, Jeong J M. Spectral Shape Tunable Bandrejection Filter Using a Long-period Fiber Grating with Divided Coil Heaters. IEEE Photonics Technology Letters 2003; 15: 407-409.

[8] Enomoto T, Shigchara M, Ishikawa S, Danzuka T, Kanamori H. Long-period Grating in a Pure-silica-core Fiber Written by Residual Stress Relaxation. OFC'98 1998; 277-278.

[9] Savin S, Digonnet M J F, Kino G S, Shaw H J. Tunable Mechanically Induced Longperiod Fiber Gratings. Optics Letter 2000; 25: 710-712.

[10] Lee N K, Song J W, Park J H. Fabrication of Fiber Device with Long-period Fiber Gratings at Locations Under Applied Pressure and its Application as Load Sensor. Japanese Journal of Applied Physics 2006; 45: 1656-1657.

[11] Wu E, Yang R C, San K C, Lin C H, Alhassen F, Lee H P, A Highly Efficient Thermally Controlled Loss-tunable Long-period Fiber Grating on Corrugated Metal Substrate. IEEE Photonics Technology Letters 2005; 17: 612-614.

[12] Lee N K, Song J W, Park J H. Mechanically Induced Long-period Fiber Grating Array Sensor 2011; 53: 2295-2298.

[13] Rego G, Fernandes J R A, Santos J L, Salgado H M, Marques P V S. New Technique to Mechanically Induce Long-period Fibre Gratings, Optical Communications 2003; 220: 111-118.

[14] Rego G. Long-period Fiber Gratings Mechanically Induced by Winding a String Around a Fiber/grooved Tube Set, Microwave and Optical Technology Letters 2008; 50: 20642068.

[15] Lee C-H, Lee J, Park J, Kim K T. Easy Fabrication of Long-period Fiber Grating by Winding a Wire around an Optical Fiber Fixed to Cylindrical Rod. Microwave and Optical Technology Letters 2012; 54: 1937-1941.

[16] Erdogan T. Cladding-mode Resonances in Short- and Long-period Fiber Grating Filters. Journal of Optical Soceity of America A 1997; 8: 1760-1773.

[17] Erdogan T. Fiber Grating Spectra. Journal of Lightwave Technology 1997; 15: 1277-1294.

[18] Gafsi R, El-Sherif M A. Analysis of Induced-birefringence Effects on Fiber Bragg Gratings. Optical Fiber Technology 2000; 6: 299-323. 